\title{
CSHP Summer Educational Sessions (SES) 2012: Poster Abstracts / Séances éducatives d'été (SÉÉ) 2012 de la SCPH : Résumés des affiches
}

CSHP 2015 is a quality program that sets out a vision of pharmacy practice excellence in the year 2015. Through this project, CSHP challenges hospital pharmacists to reach measurable targets for 36 objectives grouped under 6 goals, all aimed toward the effective, scientific, and safe use of medications and meaningful contributions to public health. CSHP 2015 applies to inpatients and outpatients, community and hospital pharmacists, and all practice settings. Posters identified with a "CSHP 2015" logo are those judged by the CSHP 2015 Steering Committee to be particularly relevant to one or more of the 36 objectives.

Le projet SCPH 2015 est un programme axé sur la qualité qui propose une vision de l'excellence en pratique pharmaceutique en l'an 2015. Au moyen de ce projet, la SCPH met les pharmaciens d'établissements au défi d'atteindre les cibles mesurables de 36 objectifs répartis entre 6 buts, visant tous l'utilisation efficace, scientifique et sûre des médicaments ainsi que des contributions significatives à la santé publique. Le projet SCPH 2015 s'applique aux patients hospitalisés et externes, aux pharmaciens d'hôpitaux et communautaires, et à tous les milieux de pratique. Les affiches marquées du logo "SCPH 2015 " sont celles que le comité directeur du projet SCPH 2015 a jugé particulièrement appropriées à l'un ou l'autre des 36 objectifs.

\section{Sunday, August 12, 2012 • \\ Dimanche 12 août 2012}

1. An Evaluation of Inpatient Diabetes Care Management among Non-Critically Ill Patients in the General Internal Medicine Unit at a Large Canadian Teaching Hospital

2. The Cost-Impact of Using Patient's Own Multi-dose Medications in Hospital

3. Integration and Evaluation of Pharmacists on the Acute Resuscitation Team

4. Rural Telepharmacy—Implementation on a Provincial Scale

5. Enhancing Customer Satisfaction with Pharmacy Services in a Province-Wide Healthcare Organization

6. Evaluation of a Fall Risk Tool Including Medication Use

7. Development, Delivery and Assessment of an Interactive Infectious Disease Educational Series for Pharmacists

8. Identification of Hospital Pharmacists' Learning Needs

9. Development of a Regional Database of Pharmacy-Based Research and Quality Improvement Projects (CSHP 2015)

\section{Monday, August 12, 2012 • Mardi 13 août 2012}

1. A Single-Center Experience of Voriconazole Therapeutic Drug Monitoring (TDM) in the Management of Leukemia/Bone Marrow Transplant (L/BMT) Patients

2. What Patients Want: Preferences Regarding Hospital Pharmacy Services (CSHP 2015)

3. Benchmarking Sedative-Hypnotic Drug Use in Nova Scotia Hospitals

4. Evaluation of Medication Turnaround Time Following Implementation of Digital Fax Technology for Prescriber Order Transmission to Pharmacy in a Tertiary Care Hospital

5. Conversion of Dialysis Patients from Epoetin Alfa to Darbepoetin Alfa is Cost-Saving in a Community Hospital Setting

6. Examining Trends in the Administration of "As Needed" Medications to Inpatients with Behavioural and Psychological Symptoms of Dementia

7. Everything Including the Lipid Sink: Emergency Department Use of Intravenous Lipid Emulsion for Amitriptyline Overdose

8. Characterization of Renal Dysfunction in a Cohort of Adult HIV-Infected Patients: A Retrospective, Observational CaseControl Study

The texts of poster abstracts are published exactly as submitted by the authors and have not undergone any copyediting by the Canadian Journal of Hospital Pharmacy.

Le Journal canadien de la pharmacie hospitalière n’a pas soumis le texte des résumés des affiches à une révision linguistique et les publie ici tels que remis par les auteurs. 


\section{An Evaluation of Inpatient Diabetes Care Management among Non-Critically III Patients in the General Internal Medicine Unit at a Large Canadian Teaching Hospital}

Andrew FW Haddy, Michael Wong, Jin-Hyeun Huh, Bohdan J Laluck Jr Toronto Western Hospital, University Health Network, Toronto, ON

Rationale: Poor management of inpatient hyperglycemia is associated with an increased risk of morbidity, mortality, length of hospital stay, and admission rate to the intensive care unit. For these reasons the American Diabetes Association, American Association of Endocrinologists and Society of Hospital Medicine recommend a fasting blood glucose target $<7.8 \mathrm{mmol} / \mathrm{L}$ and a random blood glucose target $<10.0 \mathrm{mmol} / \mathrm{L}$.

Objective: To evaluate the management of diabetes and hyperglycemia among non-critically ill patients admitted to the general medicine unit (GIM) at a large Canadian teaching hospital.

Methods: A prospective cohort study of 93 consecutive inpatients admitted to GIM at the Toronto Western Hospital was conducted. Up to 4 bedside blood glucose measurements were collected daily for 7 days following the first insulin order. The type and amount of insulin administered were also recorded. Patients receiving corticosteroids were excluded. The primary outcome was the rate of hyperglycemia $(>10 \mathrm{mmol} / \mathrm{L})$ and hypoglycemia $(<4 \mathrm{mmol} / \mathrm{L})$. Secondary outcomes were mean glucose level per patient day and proportion of insulin administered as either standing or correctional orders.

Results: Of the 93 patients, 20 (22\%) patients had at least $81 \%$ of their routine blood glucose readings above $10 \mathrm{mmol} / \mathrm{L}$ and $9(10 \%)$ patients had at least 1 reading below $4.0 \mathrm{mmol} / \mathrm{L}$. The mean rate of hyperglycemia was $58 \%$ of measurements per patient. The mean glucose level per day decreased from $12.2 \mathrm{mmol} / \mathrm{L}$ to $11.0 \mathrm{mmol} / \mathrm{L}$, over the 7-day period. In patients receiving both standing and correctional insulin, the proportion of standing insulin increased from $82.1 \%$ to $83.7 \%$ over the first 7 days.

Conclusion: Inpatient hyperglycemia is inadequately managed and improvement efforts are needed. Education strategies should focus on performing daily adjustments to basal and nutritional insulin in addition to decreasing the use of sliding scale insulin as the sole means of controlling blood glucose.

\section{The Cost-Impact of Using Patient's Own Multi-dose Medications in Hospital}

Gigi Y C Wong

Langley Memorial Hospital, Langley, BC

Rationale: The use of patient's own medications is of particular interest for its potential to minimize drug wastage and costs. However, the cost-impact is unknown when pharmacy personnel time for verification is considered.

Objectives: To determine the cost-impact of using patient's own multi-dose medications on admission in a surgical patient population as compared to routine dispensing. To describe multi-dose medications prescribing with regards to type, prevalence, and formulary status. To determine the percentage suitable for use after verification.

Study Design and Methods: This was a prospective, consecutive, case-series, time-and-motion study. Admission orders for patients newly admitted to 6 surgical units were screened to identify patient's own multi-dose medications that required verification. The total time required for all verification-related activities was captured. Data was collected over 3 weeks.
Results: Of the 250 patients admitted, $20.4 \%$ were prescribed a multidose medication. Verification was completed for 67 of 85 prescribed items, of which $91 \%$ were suitable for use. Thirty-five different medication types were identified, with $67 \%$ on formulary. The most common routes of administration were inhalation (66\%), nasal (11\%) and ophthalmic (9\%). The average cost was $\$ 24.54 \pm 32.33$ per multi-dose item. The time required for verification was $10.5 \pm 6.7$ minutes per patient $(5.2 \pm 3.3$ minutes per medication). The costimpact was calculated as the difference between the drug cost (routine hospital dispensing) and the cost of home medication verification. A one-sample t-test was conducted. The cost-impact per patient was $\$ 40.05 \pm 42.60$ (P-value $<0.001$ ) (mean $\$ 18.85 \pm 15.42$ per medication). A total cost-saving of $\$ 1601.85$ was found.

Conclusion: The cost-impact of using patient's own multi-dose medications as compared to routine dispensing resulted in a costsavings of $74 \%$, inclusive of labour costs for pharmacist verification.

\section{Integration and Evaluation of Pharmacists on the Acute Resuscitation Team}

Mark McIntyre, Michael Christian, Lisa Burry

Mount Sinai Hospital, Toronto, ON

Rationale: In Canada, the presence of a pharmacist on resuscitation teams is rare and no literature describes their participation in resuscitation scenarios or satisfaction with their involvement. We describe the process and challenges of training and integrating pharmacists on the resuscitation team at a tertiary care teaching hospital. Additionally, we report feedback from other clinicians on pharmacists' skill and value during resuscitation scenarios.

Description and Implementation: A baseline survey was given to four randomly selected pharmacists. Using the results, 8 pharmacist volunteers undertook an internal training program to review ECG interpretation, common clinical scenarios and medication preparation. The pharmacists then completed certification as Advance Cardiac Life Support (ACLS) providers. Beginning in September 2010, 2 pharmacists responded to all resuscitations on weekdays between 8 am and $4 \mathrm{pm}$. Presumed roles were as a resource for drug information and as preparers of medication.

Evaluation: Challenges identified by pharmacists were role confusion, limited hours of involvement and discomfort with the resuscitation scenario. The subjective impact of these challenges diminished as experience increased. To assess the effect of the pharmacist on the resuscitation team, a questionnaire was distributed electronically in January 2012 to 330 non-pharmacist clinicians potentially involved in the resuscitation response of which 121 responded. The most common roles of the pharmacist were to suggest dosing/administration (72\%), to prepare medications (55\%) and to suggest medication therapy (45\%). Of the 92 respondents finding the question applicable, 76\% felt that the pharmacist was very useful or somewhat useful to their role and $74 \%$ felt that the pharmacist was very useful or somewhat useful to the resuscitation response.

Practice Importance/Impact: Our experience and other clinician's feedback support the continued integration of a pharmacist on the resuscitation team. Future goals are to increase the hours of coverage and number of ACLS certified pharmacists. 


\section{Rural Telepharmacy-Implementation on a Provincial Scale}

Ian Creurer, Ryan Johnston, Doug Pankoski

Pharmacy Services, Alberta Health Services, Alberta

Rationale: Alberta Health Services (AHS) has 105 hospitals with onsite pharmacy services; 86 are rural sites with few staff, limited relief, and significant travel requirements; service consistency is a challenge. Staff absences result in dispensing activities being delayed or performed by nurses, and create gaps in pharmacist clinical activity.

Description of Practice: Telepharmacy enables offsite staff to visually verify medications prior to dispensing. Sustainable, province-wide telepharmacy became possible with formation of AHS. Previously, telepharmacy was in only five rural sites and lacked support for sustainability. The AHS rural telepharmacy model uses high definition cameras in combination with 'unified communications' (UC). UC allows desktop access to voice-over-internet, instant messaging, webbased video and document sharing within a secure environment.

Steps Taken: The telepharmacy project was a collaboration of Pharmacy Telehealth and Information Technology Project Management. Funding was provided for project management and technology; pharmacy staff were reassigned to this project. Pharmacy project staff provided all training to site-based Pharmacy staff. The first 37 sites were installed in March 2012; remaining sites will be in place by August 2012.

Evaluation: Phase one installation was completed within timelines. A pre-implementation survey captured perceptions of current dispensing systems.

Table 1: Medication Dispensing Systems Baseline Survey Results (n=128)

Satisfied with current medication dispensing system $\quad 65 \%$

Dispensing activity impacts my other responsibilities $\quad 83 \%$

I am always able to work to full scope of practice $\quad 12 \%$

I am always able to maintain service levels (53\% always/often) $\quad 19 \%$

My site has used RNs to provide med verification $\quad 29 \%$

Availability of telepharmacy would have improved dispensing $\quad 39 \%$

time in instances when no onsite Pharmacy staff were available

Availability of telepharmacy would have improved dispensing safety $\quad 41 \%$

in instances when no onsite Pharmacy staff were available

I have traveled to cover staffing shortages at other sites

Dispensing delays due to need for staff to travel have occurred

A second survey assessed satisfaction with phase one installation processes.

Table 2: Phase I installation feedback survey ( $n=34$ of 105, 32\%)

I have used the Telepharmacy webpage

There are space issues at my site with the equipment

I have used some aspect UC on a daily basis

I have used the document camera

Telepharmacy supports the ability to work according to

standards of practice

The enhanced access of peers to me is beneficial

I understand how telepharmacy could benefit me

I understand how telepharmacy could benefit

Pharmacy department

I would have preferred to be trained in person

Ratings for acceptability of the document camera

Ratings for acceptability of headsets/speakers

Importance: Advantages include: inter-site support for continuity of services despite onsite staff absences; optimized scope of practice (technician checking, pharmacist direct patient care); multi-site educational opportunities; and decreased travel for working groups.

\section{Enhancing Customer Satisfaction with Pharmacy Services in a Province-Wide Healthcare Organization}

Ian Creurer, Jeremy Slobodan

Pharmacy Services, Alberta Health Services, Alberta

Rationale: To assess customer satisfaction, Pharmacy Services conducted focus groups with nursing staff at sites across the province. An improvement initiative was selected to be applicable to all organizational settings. This was challenging, as Alberta Health Services (AHS) consists of 117,000 employees, with 103 acute and mental health hospitals and services offered at 400 facilities across Alberta. Improving awareness and access to drug information resources was selected as the improvement initiative.

Description of Program: A presentation for delivery by pharmacy staff to nursing staff was developed, describing use of online resources, the AHS Pharmacy Drug Information Service, and the role of pharmacy staff in providing drug information. A toolkit made it easier for front line pharmacy staff to organize and deliver presentations to their nursing colleagues.

Steps Taken: A staff group developed the presentation and toolkit. Toolkit items included an introductory letter to the nursing unit/site manager, tips on organizing sessions, speaker notes, sign up and evaluation forms, and handouts for attendees. Success depended on pharmacy staff taking onus to deliver the presentation to their nursing colleagues. AHS Pharmacy Leadership was engaged to promote to Pharmacy staff and to disseminate the toolkit; several site staff groups incorporated this initiative into their local Pharmacy Awareness Week activities.

\section{Evaluation:}

Table 1: Nursing Focus Group feedback - Themes

Session activity:

26 Focus group sessions at 18 hospital sites, with participation from 165 nursing staff

Themes identified:

- Increase Pharmacy Hours/ Staffing

- Improve Medication Dispensing

- Improve Process of Returning Medications to Pharmacy

- Improve Medication Ordering

- Improve Medication Storage/ Stocking at Unit

- Improve Medication Labelling

- Improve Communication

- Improve Medication Information (online and Pharmacist-provided)

- Increase Pharmacists' involvement with Patient Care

- Improve Electronic Systems

Table 2: Evaluation of "Improving Access to Drug Information" sessions Survey results

Question "Yes" responses

The duration of the training session was appropriate $99.7 \%$

I know more about which drug information resources are available $\quad 99.3 \%$

I now know better how to find the online drug information resources $99.3 \%$

I am more likely to use the online drug information resources now $\quad 98.9 \%$

I am more likely to use the AHS Drug Information Service now $\quad 98.0 \%$

The handout was helpful $\quad 98.6 \%$

I would like presentations on other topics $\quad 37.9 \%$

\begin{tabular}{lrr}
\hline & Presentation demographics \\
\hline Date range & June 2011 to March 2012 \\
\# Presentations & 102 \\
\# Sites participating & 48 \\
\# Nurses participating & 1121 \\
Survey responses (rate) & $767(68 \%)$ \\
\hline
\end{tabular}


Importance: This addressed a nurse-identified need, to support better patient care. In addition, interaction and dialogue was promoted between front line pharmacy and nursing staff.

\section{Evaluation of a Fall Risk Tool Including Medication Use \\ Lisa Currie ${ }^{l}$, Katherine Giannoulis', Ingrid Sketris', Gordon Flowerdew', Patricia Bilski², Kathy MacPherson', Graeme Bethune ${ }^{2}$ \\ ${ }^{1}$ Dalhousie University, Halifax, NS \\ ${ }^{2}$ Capital District Health Authority, Halifax, NS}

Rationale: Falls cause significant morbidity and mortality in the elderly and medications contribute to falls risk. Various tools have been used to assess risk. The Veterans' Memorial Building (VMB) a longterm care facility in Halifax uses the Fall Risk Tool (FRT) (adapted from Oak Brook Healthcare Centre) which assesses 8 domains including medications.

Objectives: To examine the association between the Fall Risk Tool including medication use and falls in residents at the VMB.

Methods: All individuals admitted to the VMB within a 16 -month period (January 2009-April 2010) $(\mathrm{N}=132)$ and who had a FRT completed were assessed $(\mathrm{N}=76)$. Electronic incidence charts provided fall occurrence data. Drug use was obtained from pharmacy dispensary records. Drugs were assigned WHO-Anatomical Therapeutic Chemical 2012 codes and classified by fall risk category determined by literature. Descriptive statistics, Kaplan-Meier curves and proportional hazards analysis were used to determine if the FRT score and drug use were predictive of subsequent falls.

Results: 76 patients had an FRT completed. FRT scores ranged from 2-20 with a mean value of 11.51 (SD 4.19). Most residents (59.2\%) demonstrated a low risk with a score between 0-12 and did not have a documented fall occurrence (59.21\%). 97.5\% of patients were prescribed at least one high risk medication. Of the 3207 drugs prescribed a high proportion $(66.9 \%)$ were classified as low risk medications. Of all medications prescribed, the most frequently prescribed class of high risk medication was narcotics (5.5\%).

Conclusion: Most VMB residents (97.5\%) received at least 1 high risk medication associated with falls. Pharmacists should be familiar with falls risk assessment tools. A high score on the medication domain may serve as a useful prompt for a medication review. Work is needed to determine how to improve early assessment of fall risk and to decrease fall occurrences via implementation of prevention strategies.

\section{Development, Delivery and Assessment of an Interactive Infectious Disease Educational Series for Pharmacists}

\section{Duclos, R Law}

St Michael's Hospital, Toronto, ON

Rationale: The framework for our antimicrobial stewardship program emphasizes that all clinicians are responsible for antimicrobial stewardship, and identifies education as a core component of ASP. To support our pharmacists in being stewards, the Infectious Disease (ID) Educational Series for Pharmacists was developed, implemented and assessed.

Description: Topics including interpreting and utilizing antibiograms, antimicrobial de-escalation, antimicrobial pharmacokinetics and pharmacodynamics, intravenous to oral conversion of antimicrobials were presented in 13 bi-monthly sessions. Content included foundational ID knowledge and specific examples of how to optimize antimicrobial therapy. Presentations were designed around a game show format to engage the audience and provide opportunities for participants to solve case-based questions. Participants completed a three question pre- and post-test to assess content knowledge as well as a presentation evaluation, which utilized a 5-point scale to assess content, delivery, and visuals and materials.

Evaluation: A total of 14.5 hours of content was delivered with an average of 18 (range 12 to 23) participants attending each session. The percentage of participants who had a perfect score in the post-test was $68 \%$ compared to $40 \%$ in the pre-test $(\mathrm{p}<.0001$ ), suggesting an increase in ID knowledge. All respondents rated content, delivery, and visuals and materials in the range of 'acceptable' to 'excellent', with a median score (out of 5) of 5 for content, 5 for delivery and 4 for visuals and materials. Based on the written comments, the content and format of the presentations were positively received and topics covered were useful for practice.

Conclusion: The interactive ID educational series was well-received by participants and enhanced pharmacists' ID/antimicrobial stewardship knowledge to support their role as antimicrobial stewards.

\section{Identification of Hospital Pharmacists' Learning Needs}

Stacey MacAulay', Jennifer Ryan', Douglas Doucette

${ }^{1}$ Horizon Health Network, Moncton, NB

${ }^{2}$ Horizon Health Network, Saint John, NB

Objective: In 2008, New Brunswick Department of Health reorganized from 8 to 2 regional health authorities (RHAs). One of these 2 resulting RHAs, Horizon Health Network, is now the largest health authority in Atlantic Canada and currently employs 102 pharmacists. There is substantial variation among these pharmacists' practice sites, their educational degrees and speciality training, and their percentage of time spent in direct patient care, centralized services, and research and education activities. Therefore, a survey was conducted to identify the learning needs of this diverse group of pharmacists, for the purpose of influencing the development of a 3-year education plan.

Objectives: The primary objective of this survey was to identify the learning needs of pharmacists at Horizon Health Network. The secondary objective was to determine the preferred characteristics of continuing education sessions.

Methods: The study population was all pharmacists employed at Horizon Health Network. The survey was administered electronically and contained the categories of demographics, patient care, teaching, research, therapeutics, CSHP 2015, pharmacist prescribing, and characteristics of continuing education sessions.

Results: The response rate was $77.5 \%$. Evidence-based practice, teaching skills, and research skills were the main learning needs identified regarding skill development. The therapeutic topics that were most commonly identified as learning needs were anticoagulation and infectious diseases. Learning needs related to pharmacist prescribing included: 1) ordering and interpreting lab tests; 2) performing therapeutic drug monitoring; and 3) implementing a communication system to notify physicians of pharmacists' involvement. Out of the 10 CSHP 2015 objectives addressed in the survey, there was only 1 for which $\geq 50 \%$ of pharmacists felt either highly or very highly prepared. The preferred frequency for continuing education sessions was once monthly, with case presentations being the preferred format.

Conclusion: Pharmacists identified a wide variety of learning needs relating to skill development and knowledge advancement. 


\section{Development of a Regional Database of Pharmacy- Based Research and Quality Improvement Projects}

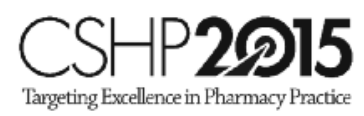

Stacey MacAulay', Jennifer Ryan', Douglas Doucette

${ }^{1}$ Horizon Health Network, Moncton, NB

${ }^{2}$ Horizon Health Network, Saint John, NB

Rationale: In 2008, New Brunswick Department of Health reorganized from 8 to 2 regional health authorities (RHAs). One of these 2 resulting RHAs, Horizon Health Network, is now the largest health authority in Atlantic Canada and employs 102 pharmacists and 159 pharmacy technicians. Given the large size of this pharmacy team, a mechanism was needed to track ongoing projects. Therefore, a survey was conducted to identify recent and ongoing pharmacy-based research and quality improvement projects being undertaken throughout the RHA. A database of these projects was then developed.

Objectives: The primary objective of this survey was to identify pharmacy-based research and quality improvement projects completed within the last 5 years at Horizon Health Network, for incorporation into a regional database.

Methods: The study population was all pharmacists and pharmacy technicians employed at Horizon Health Network. They were contacted via email and asked to complete a questionnaire. The questionnaire sought to obtain general information about the project, members of the research team, project status (i.e. complete/ incomplete), research funding, and posters or oral presentations completed.

Results: A total of 61 projects was submitted, 48 of which were classified as research projects and 13 of which were quality improvement projects. The most common study design was a prospective cohort study. Medication/patient safety was the most common focus of the projects, followed by the value/role of a pharmacist. The database was developed using Microsoft Access and will be updated periodically. Anticipated uses of the database include assisting with preparing reports for management teams, identifying research themes and resource gaps, identifying staff members involved with research, assisting with project timeline management, and facilitating collaboration among staff.

Conclusion: A regional database was successfully developed which contains searchable information on numerous research and quality improvement projects conducted within Horizon Health Network within the past 5 years.

\section{A Single-Center Experience of Voriconazole Therapeutic Drug Monitoring (TDM) in the Management of Leukemia/Bone Marrow Transplant (L/BMT) Patients}

Janice Yeung', Yu-Chen Lin', Dailin Li², Morris Pudek², Diane Roscoe ${ }^{2}$, Carmen Mountford ${ }^{1}$, Trana Hussaini ${ }^{1}$, Maryse Power ${ }^{3}$, Raewyn Broady ${ }^{3}$ ${ }^{1}$ CSU Pharmaceutical Sciences, Vancouver, BC

${ }^{2}$ Pathology \& Laboratory Sciences, Vancouver, BC

${ }^{3}$ Leukemia/BMT Program of BC, Vancouver, BC

Background: Voriconazole is frequently used in the prevention and treatment of fungal infections in immunocompromised patients. Nonlinear pharmacokinetics and wide inter- and intrapatient variability lead to unpredictable plasma concentrations. Studies demonstrate that voriconazole trough levels correlate with efficacy and toxicity and it is proposed that TDM may improve patient outcomes. A 6-month pilot project was initiated to evaluate the appropriateness and ordering and sampling practices of voriconazole TDM in our patients.

Methods: All TDM episodes performed from May 1 to November 30, 2011 at the L/BMT Program of British Columbia were included. Voriconazole was measured by UPLC using a UV detector. Steady-state voriconazole trough levels were defined as the following: undetectable (less than $0.3 \mathrm{mg} / \mathrm{L})$; well below therapeutic range $(0.3-0.7 \mathrm{mg} / \mathrm{L})$; borderline therapeutic $(0.8-0.9 \mathrm{mg} / \mathrm{L})$; therapeutic $(1-5.5 \mathrm{mg} / \mathrm{L})$; toxic (greater than $5.5 \mathrm{mg} / \mathrm{L}$ ).

Results: 59 patients were included and 290 levels were performed. Initial voriconazole concentrations were non-therapeutic in 26/59 patients: 2 undetectable, 12 well-below therapeutic, 5 borderline therapeutic and 7 toxic. Of these 26 patients, only 6 had their dosage adjusted and 20 did not. Reasons for non-action included inappropriately drawn levels, not at steady-state, prophylaxis, desire to wait for a repeat level or no reason was given at all. Of those 6 patients where an action was taken, 3 became therapeutic, 1 borderline therapeutic and 2 did not have follow-up levels.

Conclusion: This pilot study highlights the importance of appropriate TDM sampling and interpretation. 26/59 (44\%) patients had initial voriconazole levels that were not therapeutic and only 6 of those patients had their voriconazole dosing adjusted. On the basis of these findings, we conclude that voriconazole TDM should be implemented to optimize patient outcomes and that there is a need for a standardized dosing adjustment algorithm to assist clinicians in interpreting and adjusting dosing for voriconazole trough levels.

\section{What Patients Want: Preferences Regarding Hospital Pharmacy Services}

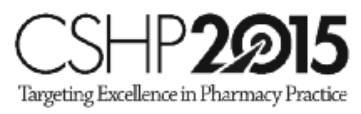

Douglas Doucette', Paula Buckley', Odette Gould ${ }^{2}$

${ }^{2}$ Regional Pharmacy Services, Horizon Health Network, New Brunswick ${ }^{2}$ Department of Psychology, Mount Allison University, Sackville, NB

Rationale: The role of hospital pharmacists has evolved over the past couple decades from medication preparation and distribution to active involvement in health teams as clinicians who identify and resolve patients' medication-related issues in an effort to improve patient outcomes. As part of a phone survey to determine the baseline prevalence of patients who recall interacting with the pharmacist during their hospital admission (CSHP 2015 Objective 1.5), we also asked about their preferences for pharmacy services provided.

Objectives: To analyse content of open-ended survey responses to the question asked of patients recently discharged from hospital.

Methods: A telephone questionnaire was completed by 400 former inpatients randomly selected following discharge from acute care hospitals. Responses were recorded to the question "what service or information would you like a pharmacist to provide in the hospital that would most help you in managing your medications?" Two raters established response categories and independently scored the survey responses. Inter-rater agreement was measured for number of items present in a participant's response (segmentation) and how to categorize each item identified (content categorization).

Results: Three global categories of responses were obtained, with each having multiple sub-categories: Information about Medication; SelfDisclosure, and Pharmacy Services. Almost half of all responses $(\mathrm{n}=192)$ were related to general information about medication, e.g. list, 
purpose, new meds. Other frequently used sub-categories were SelfDisclosures ( $\mathrm{n}=142$; experience with pharmacy, medication or hospital) and Medication Cautions ( $\mathrm{n}=139$; side effects, interactions, allergies). Responses were less frequently under sub-categories of Adherence $(n=67)$, Services (specific to Pharmacy, $n=49$ ), or Information Source (regarding medications, $\mathrm{n}=36$ ). Total agreement rate for segmenting was $92.49 \%$ and for content categorization was $85.86 \%$.

Conclusions: Most patients would like a hospital pharmacist to provide a general medication overview, including information about side effects and interactions, during their admission. A future study may assess patients' willingness to select from a guiding list of potential clinical services.

\section{Benchmarking Sedative-Hypnotic Drug Use in Nova Scotia Hospitals}

$S$ Bowles ${ }^{l}, H$ Neville ${ }^{l}, J$ Dagenais
${ }^{1}$ Capital Health Pharmacy Department, Halifax, NS
${ }^{2}$ University of Toronto, Toronto, ON

Background: The use of sedative-hypnotic drugs (SHD) is associated with falls in the elderly which can lead to disability, hospitalization, and death. The use of SHD in Nova Scotia (NS) hospitals is unknown.

Objectives: To evaluate the magnitude and type of SHD used in nine provincial district health authorities.

Study Design and Methods: This was a retrospective evaluation of SHD use (benzodiazepines, chloral hydrate, trazodone and zopiclone) for the years 2009-2011 in adult hospitals. Hospital purchasing data was obtained from the NS Provincial Drug Distribution Program. Data was converted to defined daily doses (DDD) and divided by 100 patient-days for each hospital. Hospitals were grouped by district and by size and median use analyzed using the Kruskal-Wallis test. Pharmacy directors were provided with confidential reports to benchmark their district against the provincial rate.

Results: Eight of 15 SHDs accounted for $96 \%$ of total use. The overall provincial rate was $51.7 \mathrm{DDD} / 100$ patient-days; individual district rates ranged from $34.3-61.8$. There was no significant difference in median use among the nine districts $(\mathrm{p}=0.16)$. The most frequently used SHDs were zopiclone (15.4 DDD/100 patient-days), lorazepam (14.3), and diazepam (8.6). No differences in use of SHDs were observed based on hospital size. Pharmacy directors found the benchmarking method to be useful and thought that having the information would improve the quality and safety of patient care.

Conclusion: Benchmarking sedative-hypnotic drug use can be a useful indicator of overall use, identifies the most common SHDs, and provides preliminary data to support more in-depth analysis of the appropriate use of SHDs in NS hospitals. Other institutions can compare their own usage against the NS standardized rate.

\section{Evaluation of Medication Turnaround Time Following Implementation of Digital Fax Technology for Prescriber Order Transmission to Pharmacy in a Tertiary Care Hospital}

\section{Nodwell', H Neville', S Alsharif}

${ }^{1}$ Capital Health, Halifax NS

${ }^{2}$ Dalhousie University, Halifax NS

Rationale: Reducing medication turnaround time in hospitals can improve efficiency, patient safety and quality of care. Scanning Digital Physician Order Technology (SDPOT), which scans physician orders by nursing staff and sends them digitally to the pharmacy for verification and processing, was implemented to reduce turnaround time.

Objectives: To evaluate medication turnaround time before and after implementation of SDPOT for all medications and antibiotics only.

Study Design and Methods: This was a retrospective evaluation of the time between physician composition and pharmacy verification of orders (Phase 1) and the time between physician composition to nurse administration of the medication to the patient (Total). Medication orders were audited during June 6-10, 2011 (before) and September 26-30, 2011 (after) for three hospital sites. Included orders were composed while the pharmacy was open, composed and administered on the same day, new orders or an increase in dose, frequency or change in route. For medication order sets, only the first order was included. Prescriptions that were PRN (as needed), self-administered, or chemotherapy were excluded. Median turnaround times were analyzed in SPSS using the Mann-Whitney U test.

Results: There were 356 orders and 304 orders audited before and after SDPOT implementation, respectively. The primary reason for excluding orders was lack of a prescriber composition time. The median turnaround time for Phase 1 for all medications was $2 \mathrm{~h} 13 \mathrm{~min}$ before SDPOT, which significantly decreased to $1 \mathrm{~h} 45 \mathrm{~min}$ after SDPOT $(\mathrm{p}=0.03)$. Total turnaround time also significantly decreased from $5 \mathrm{~h}$ $29 \mathrm{~min}$ to $4 \mathrm{~h} 59 \mathrm{~min}$ for all medications $(\mathrm{p}=0.04)$. There were no differences in turnaround times for antibiotics.

Conclusion: Implementation of SDPOT was associated with a decreased medication turnaround time of 30 minutes. Other health information technology solutions, such as computerized physician order entry, should be explored to further decrease turnaround times.

\section{Conversion of Dialysis Patients from Epoetin Alfa to Darbepoetin Alfa is Cost-Saving in a Community Hospital Setting}

C Awde-Sadler, J Kirkwood, E Beaubien

Peterborough Regional Health Centre, Peterborough, $\mathrm{ON}$

Rationale: Erythropoiesis-stimulating agents (ESA), epoetin alfa (EPO) and darbepoetin alfa (DPO), manage anemia in hemodialysis (HD) patients.

Objectives: To compare patient stability and the cost of EPO vs. DPO in a community HD clinic.

Methods: Patients at the Peterborough Regional Health Centre (PRHC) were switched from EPO to DPO according to the dose conversion ratio (DCR) used at a teaching hospital (The Ottawa Hospital). Individual doses were adjusted to maintain patients' target hemoglobin $(\mathrm{Hb})$. All patients for whom data were available on the ESA dose for one month before and 6 months after the switch were included in this retrospective study. Weekly doses of ESA, and monthly $\mathrm{Hb}$, ferritin, and transferrin saturation (TSat) were recorded. Paired sample signed rank tests were performed.

Results: The mean age of patients $(n=50)$ was 71 years. Median weekly doses of EPO and DPO were $8000 \mathrm{IU}$ and $28 \mathrm{mcg}$, respectively [median DCR $=286]$. Mean $\mathrm{Hb}$ and ferritin remained stable with the switch from EPO to DPO [Hb: 114 and $111 \mathrm{~g} / \mathrm{L}$ ( $\mathrm{p}=0.0538$ ); ferritin: 497 and $521 \mathrm{mcg} / \mathrm{L}(\mathrm{p}=0.29)$, respectively]. There was a small drop in the mean TSat from $31 \%$ to $24 \%(\mathrm{p}=0.0001)$. Assuming list prices of $\$ 0.01425 / \mathrm{IU}$ EPO and $\$ 2.68 / \mathrm{mcg}$ DPO, the average costs of ESA per patient were $\$ 114$ and $\$ 75$, respectively. An average of $\$ 39 /$ week/patient was saved with DPO. With over 200 patients treated annually, >\$405,600 could be saved each year at the PRHC. 
Conclusion: This "real-world" cost-effectiveness evaluation of community hospital HD patients switched from EPO to DPO showed the potential for significant cost-savings without negatively impacting target anemia parameters. The results were consistent with those previously reported from larger teaching centres despite the more elderly population in this community setting indicating that an EPO:DPO DCR > 200 and substantial ESA cost savings may be achieved in most HD settings.

\section{Examining Trends in the Administration of "As Needed" Medications to Inpatients with Behavioural and Psychological Symptoms of Dementia}

Robert Neumann', Peter Faris', Janise Proudfoot'

${ }^{1}$ Centennial Centre for Mental Health and Brain Injury, Ponoka, $A B$

${ }^{2}$ Foothill Medical Centre, Calgary, $A B$

Rationale: The use of chemical restraint on a "pro re nata" (PRN) basis is utilized in patients suffering from behavioural and psychological symptoms of dementia (BPSD), but may lead to double dosing and adverse effects.

Objectives: To identify individuals who were predisposed to PRN administration and at which times PRN restraint was more likely to be administered.

Methods: Chart reviews were completed on patients who had a diagnosis of dementia from May 1, 2009 until April 30, 2011. Medication administration records were reviewed for PRN use of medications prescribed for aggression, agitation, or insomnia (antipsychotics, benzodiazepines, antidepressants, mood stabilizers, zopiclone). Data collected included age, sex, time of day, and day of week of administration. Poisson regression models were used to model associations between factors of interest and rates of PRN use.

Results: A total of 170 individuals with dementia were included; $49 \%$ were males and $42 \%$ of patients were 65-79 years. Over a total of 49221 bed days 3958 PRNs were administered. Rates of PRN use were over twice as high in patients $<65$ years when compared with patients $>=80$ years (rate ratio $[\mathrm{RR}]: 2.26 ; 95 \%$ Confidence Interval $[\mathrm{CI}]=$ 1.21-4.24). Also, rates of PRN administration were higher between $1600-1700(\mathrm{RR}=2.94 ; 95 \% \mathrm{CI}=2.44-3.74)$ and $0000-0100(\mathrm{RR}=$ 2.57; $95 \% \mathrm{CI}=2.04-3.22)$ compared to average rates. Weekends had a $21 \%$ higher rate of administration compared to weekdays (95\% CI: 17\%-26\%).

Conclusions: Younger individuals with dementia were more likely to receive PRN medications for BPSD. In addition, PRNs were more likely to be administered on weekends, and at particular times during the day. Sundowning, staff shift change and administration in lieu of adequate staffing may explain some of the differences in rates of administration, but further research is needed to evaluate the outcomes associated with increased use. The temporal rates may be explained by sundowning and shift change, while the higher rates on weekends, may be explained by other environmental factors including administration in lieu of adequate staffing.

\section{Everything Including the Lipid Sink: Emergency Department Use of Intravenous Lipid Emulsion for Amitriptyline Overdose}

Sally Ginson Duke, Leslie Manuel

Horizon Health Network, The Moncton Hospital, Moncton, NB

Rationale: Existing acute management guidelines for tricyclic antidepressant overdose do not include intravenous lipid emulsion (ILE) as a standard intervention. References suggest to consider ILE use in the event of refractory hypotension or dysrhythmias.

Description of Case: A 50 year old male arrived unresponsive at the emergency department. Electrocardiogram (ECG) reflected a wide complex tachycardia and a tricyclic overdose was suspected. A pharmacy fax confirmed amitriptyline $25 \mathrm{mg}$ x 200 tablets had been filled on the date of presentation and this was believed to be the only medication ingested. Cardiac arrest ensued and generalized seizure activity observed. Sodium bicarbonate boluses and infusion were initiated in addition to fluid resuscitation, dopamine and norepinephrine intravenous (IV) infusions, and single IV doses of midazolam and phenytoin. Despite these measures, recorded blood pressure (BP) averaged 70/39 with widened QRS complex intermittently noted via ECG. Decision made to administer ILE $20 \%$ at dose of $100 \mathrm{ml} \mathrm{IV}$ over 1 minute then $400 \mathrm{ml}$ IV over 30 minutes. Minutes after ILE administration, BP increased to $85 / 50$. Four hours following ILE, the dopamine infusion was stopped and norepinephrine weaned to a maintenance rate. Patient remained hemodynamically stable thereafter. Evaluation of Literature: The use of ILE is a relatively new intervention in the management of tricyclic overdose. At least 6 published case reports in adults and 1 published case report in a toddler describe ILE use as beneficial in significant overdoses of tricyclic antidepressants.

Importance of Case to Pharmacy Practitioners: Sharing this case will add to the current literature describing use of ILE, the benefit of which may outweigh any risk in a tricyclic overdose not responding to conventional treatment. Emergency departments should stock the $500 \mathrm{ml}$ bags of $20 \%$ lipid emulsion with other antidotes in an easily accessible location.

\section{Characterization of Renal Dysfunction in a Cohort of Adult HIV-Infected Patients: A Retrospective, Observational Case-Control Study}

M MacNeil', B Lynn Johnston ${ }^{2,3}$, P Poyah ${ }^{2,4}$, D Haase ${ }^{2,3}, K_{\text {Thompson }}^{2}$, KS Slayter ${ }^{1,2,3}$

${ }^{1}$ Department of Pharmacy, Capital District Health Authority, Halifax, NS ${ }^{2}$ Department of Medicine, Dalhousie University, Halifax, NS

${ }^{3}$ Division of Infectious Diseases, Capital District Health Authority, Halifax, NS

${ }^{4}$ Division of Nephrology, Capital District Health Authority, Halifax, NS

Rationale: The incidence and prevalence of kidney disease are rising among HIV-infected individuals. Limited knowledge is available on how to best identify and then subsequently manage those patients deemed to be at risk for renal dysfunction or damage.

Objectives: The primary objective of our study was to identify risk factors for renal dysfunction and/or renal damage. Our secondary objective was to develop a tool that could identify and manage those HIV-infected patients at increased risk for renal dysfunction. 
Study Design and Methods: This was a retrospective, observational, case-control study comparing HIV-infected patients with renal dysfunction and/or damage to those without dysfunction/damage. Renal dysfunction was defined as a Glomerular Filtration Rate (GFR) $<60 \mathrm{ml} / \mathrm{min}$. Renal damage was defined as the presence of microalbuminuria. Chi square tests and subsequent multivariate analysis were used to analyze relationships between study groups.

Results: 72-patients with renal dysfunction and/or damage were compared to 105 controls. Factors shown to be associated with renal dysfunction and/or damage included the use of non-antiretroviral medications $(55.6 \%$ vs. $34.3 \%, \mathrm{P}=0.0050)$; hypertension $(43.1 \%$ vs. $20 \%, P=0.0009)$, moderate-to-severe liver disease $(4.2 \%$ vs. $0 \%$,
$\mathrm{P}=0.0656)$, diabetes (with or without end-organ damage $(6.9 \%$ vs. $1 \%, \mathrm{P}=0.0415 ; 15.3 \%$ vs. $3.8 \%, \mathrm{P}=0.0071)$ ), and nadir CD4 cell count (182 vs. $220, \mathrm{P}=0.0769)$. After multivariate analysis, hypertension $(\mathrm{OR}=2.28 ; \mathrm{p}=0.0332)$ remained as an independent risk factor for renal dysfunction or damage.

Conclusions: Our study demonstrates the impact that chronic disease states have on the risk for developing renal dysfunction and/or renal damage in patients infected with HIV. A tool for identifying and managing patients with early signs of renal dysfunction was developed that stresses the importance of both chronic disease management and the appropriate monitoring of potentially nephrotoxic medications.

\section{ON THE FRONT COVER}

\section{Fishing Harbour, Prince Edward Island}

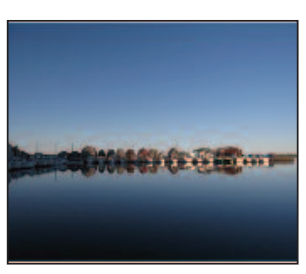

CSHP member Natalie Garwah and her family came across Malpeque Harbour on their first night of exploring Prince Edward Island in June 2010. After 7 consecutive days of rain during their road trip, it was finally a perfect evening: sunny, clear, and calm. The picturesque harbour represented a great photo opportunity for Natalie's fishing boat-obsessed husband, Claude. So, while the kids ate ice cream in the back seat, he stepped out to take this picture, using the family's Canon SD 880IS camera. "The camera may be small, but it has given us some amazing shots during our travels throughout Canada," says Natalie. "This particular photo, mounted on our living room wall, is a wonderful reminder of our East Coast trip."

The CJHP would be pleased to consider photographs featuring Canadian scenery taken by CSHP members for use on the front cover of the journal. If you would like to submit a photograph, please send an electronic copy (minimum resolution $300 \mathrm{dpi}$ ) to Colleen Drake at cdrake@cshp.ca. 